\title{
Adapting the Full Matrix Capture and the Total Focusing Method to laser ultrasonics for remote non destructive testing
}

\author{
$1^{\text {st }}$ Theodosia Stratoudaki \\ Department of Electronic and Electrical Engineering \\ University of Strathclyde \\ Glasgow, UK \\ t.stratoudaki@strath.ac.uk
}

\author{
$3^{\text {rd }}$ Paul D. Wilcox \\ Department of Mechanical Engineering \\ University of Bristol \\ Bristol, UK \\ p.wilcox@bristol.ac.uk
}

\author{
$2^{\text {nd }}$ Matt Clark \\ Department of Electrical and Electronic Engineering \\ University of Nottingham \\ Nottingham, UK \\ matt.clark@nottingham.ac.uk
}

\begin{abstract}
Laser Induced Phased Arrays (LIPAs) use post processing to focus and steer the laser generated and detected ultrasonic beam, synthesizing a phased array. The technique is broadband, non-contact, and couplant free, making LIPAs suitable for large stand-off distances, inspection of components of complex geometries and hazardous environments. This paper presents LIPAs synthesized by capturing the Full Matrix (FMC), at the nondestructive, thermoelastic regime. The Total Focusing Method (TFM) is used as the imaging algorithm, where the captured signals are summed with the appropriate time delay, in order to synthesize a focus at every point in the imaging area. The FMC and the TFM, are adapted to the needs of LIPAs in order to enable fast imaging and make more efficient use of the information in the data. Experimental results are presented from nondestructive, laser ultrasonic inspection of an aluminum sample with side drilled holes at depths varying between 10 and $20 \mathrm{~mm}$ from the surface.

Index Terms-laser ultrasonics, phased arrays, remote sensing, nondestructive testing
\end{abstract}

\section{INTRODUCTION}

Laser Induced Phased Arrays (LIPAs) use laser based ultrasound principles in order to generate and detect and post processing in order to focus and steer the generated ultrasonic beam, synthesizing a phased array [1]. A short pulsed laser of nanoseconds duration is focused on the surface of the component under test and a broadband signal is generated, which is detected by a broadband interferometer. The energy density of the generation laser is kept below the damage threshold of the material, operating at the safe thermoelastic regime and the technique is non destructive. Bulk and surface waves are excited and detected simultaneously and the technique is remote, lending itself to applications in extreme environments [2]. Due to the very small footprint of the laser beams - less than a millimeter- and the fact that the lasers can be fiber coupled, the technique could address complex geometries [3] and places of restricted access [4].
In the results presented, the data acquisition method known as the Full Matrix Capture (FMC) is used, where all possible transmitter-receiver combinations in the array are obtained by scanning the generation and detection laser beams. Following the FMC, the Total Focusing Method (TFM) is used as the imaging algorithm for the data. In the TFM, the captured signals are summed using the appropriate time delay, in order to synthesize a focus at every point in the imaging area.

The FMC and TFM are methods developed for transducer based phased arrays but LIPAs have significant differences from them (broadband, multi-mode generation/detection, directivity pattern). The aim of the present paper is to adapt the FMC and the TFM to the needs of LIPAs in order to make more efficient use of the information in the data.

\section{THEORY}

The laser beam of the pulsed laser used for ultrasonic generation in our experiments, was focused onto a line. It generated a thermoelastic source at the surface of the irradiated component, due to the absorbed laser energy. The directivity patterns for the generated longitudinal and shear waves are given by [5], [6] and using their expressions for directivity of longitudinal and shear waves, for the case of aluminum (longitudinal velocity $\sim 6400 \mathrm{~ms}^{-1}$, shear velocity $\sim 3200$ $\mathrm{ms}^{-1}$ ), it is found that the directivity pattern of the longitudinal waves has its maxima at $\theta= \pm 66^{\circ}$ and for shear waves the maxima are at $\theta= \pm 30^{\circ}$.

A laser vibrometer, sensitive to the out-of-plane ultrasonic component, was used for signal detection. Its sensitivity to longitudinal and shear waves as a function of incident wave function is given by [7]. For the case of aluminum, the sensitivity pattern for the longitudinal waves displays a uniform response with respect to angle, while the sensitivity for the shear waves has maxima at $\theta= \pm 36^{\circ}$. 


\section{The Full Matrix CAPTURE AND the Total FOCUSING METHOD}

The generation directivity and sensitivity patterns for laser generation and detection of ultrasound, differ and it is expected that this will affect the imaging performance of the LIPA. In order to predict the FMC data set, a model is used which allows the individual signals $h_{g d}(t)$ to be predicted, where the indices $g$ and $d$ refer to ultrasonic generation and detection positions respectively, and assess the experimental results. It is a forward, ray based model, that simulates the response of the system to one or more small targets and only first order scattering is considered in the case of multiple targets. There are four responses from each target, corresponding to all possible combinations for generation and detection with respect of the mode of the wave: two bulk wave modes (longitudinal-longitudinal and shear-shear) and two converted wave modes (longitudinal-shear and shear-longitudinal). These are treated separately and superposed. In the frequency, $\omega$, domain, the response to the $j^{\text {th }}$ target for generated mode, $\alpha=\mathrm{L}$ or $\mathrm{T}$ (L for longitudinal and $\mathrm{T}$ for shear), and detected mode, $\beta=\mathrm{L}$ or $\mathrm{T}$, can be written as [1]:

$$
\begin{aligned}
& H_{g d j}^{\alpha \beta}(\omega)=\frac{G_{\alpha}\left(\theta_{g j}\right) D_{\beta}\left(\theta_{d j}\right)}{\left(\left|\mathbf{d}_{\mathbf{g} \mathbf{j}}\right| \mathbf{d}_{\mathbf{d j}} \mid\right)^{1 / 2}} \exp \left[-i \omega\left(\frac{\left|\mathbf{d}_{\mathbf{g j}}\right|}{c_{\alpha}}+\frac{\left|\mathbf{d}_{\mathbf{d j}}\right|}{c_{\beta}}\right)\right] \\
& \times A_{j}^{\alpha \beta}\left(\theta_{g j}, \theta_{d j}, \omega\right)
\end{aligned}
$$

where $G_{\alpha}$ and $D_{\beta}$ describe the directivity and sensitivity patterns, $\theta_{g j}$ and $\theta_{d j}$ are the angles (relative to the surface normal) of the rays between the generation and detection positions and the target, $\mathbf{d}_{g j}$ and $\mathbf{d}_{d j}$ are the corresponding path lengths (see Fig. 1), and $A_{j}^{\alpha \beta}\left(\theta_{g j}, \theta_{d j}, \omega\right)$ is the angulardependent response or scattering matrix [8] of the target.

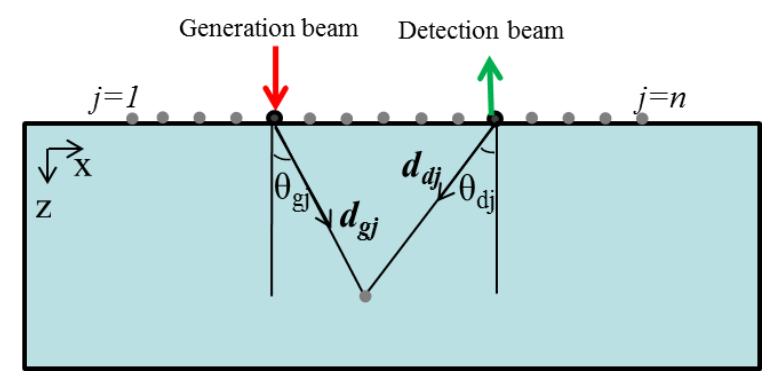

Fig. 1. Schematic diagram showing angles and path lengths, using the notation for TFM.

The imaging algorithm used in this study was the TFM, where the signals from all elements of the LIPA are summed in order to synthesize a focus at every point in the imaging area. Here, the angular dependence of the generation and detection patterns is taken into account by introducing apodization at the imaging algorithm: the contribution of each waveform is weighted by the transmit and receive directivity functions at each image point. If noise in the original waveforms is uniform then this apodization maximizes the signal to noise ratio (SNR) at each point in image. As a result, the intensity of the image, $I(r)$, is given by [9], [10]:

$$
I(\mathbf{r})=\left|\sum_{g=1}^{n} \sum_{d=1}^{n} Z_{g}(\mathbf{r}) Z_{d}(\mathbf{r}) s_{g d}\left(t_{g d}(\mathbf{r})\right)\right|
$$

where the double summation is over all combinations of ultrasonic generation $(g)$ and detection $(d)$ positions. The signal $s_{g d}(t)$ is the digitally filtered time-traces of the raw signals collected during the experiment and the time delay term $\left(t_{g d}\right)$ is given by:

$$
t_{g d}=\frac{d_{g}(\mathbf{r})+d_{d}(\mathbf{r})}{c_{T}}
$$

where $d_{g}(\mathbf{r})$ and $d_{d}(\mathbf{r})$ are the distances associated with the generation and detection ray-paths to point $\mathbf{r}$. $Z_{g}$ and $Z_{d}$ are apodization coefficients:

$$
\begin{aligned}
& Z_{g}(\mathbf{r})=\frac{G_{\alpha}\left(\theta_{g}(\mathbf{r})\right)}{\left[d_{g}(\mathbf{r})\right]^{1 / 2}} \\
& Z_{d}(\mathbf{r})=\frac{G_{\beta}\left(\theta_{d}(\mathbf{r})\right)}{\left[d_{d}(\mathbf{r})\right]^{1 / 2}}
\end{aligned}
$$

The response model can be combined with the revised TFM description to produce, what is defined as, a sensitivity image, which describes the amplitude expected from a perfect point target $\left(A_{j}^{\alpha \beta}\left(\theta_{g j}, \theta_{d j}, \omega\right)=1\right)$ as a function of position. For example, in the case of the shear - shear mode combination, the sensitivity image is given by:

$$
E(\mathbf{r})=\left|\sum_{g=1}^{n} Z_{g}(\mathbf{r}) \frac{G_{T}\left(\theta_{g}(\mathbf{r})\right)}{\left[d_{g}(\mathbf{r})\right]^{1 / 2}} \sum_{d=1}^{n} Z_{d}(\mathbf{r}) \frac{D_{T}\left(\theta_{d}(\mathbf{r})\right)}{\left[d_{d}(\mathbf{r})\right]^{1 / 2}}\right|
$$

Finally, the normalized image is introduced, which defined as the ratio of the TFM image over the sensitivity image:

$$
N(\mathbf{r})=\frac{I(\mathbf{r})}{E(\mathbf{r})}
$$

The normalized image has uniform sensitivity but non-uniform noise, as the noise in areas of weak signak is amplified.

\section{EXPERIMENTAL RESULTS}

\section{A. Experimental setup}

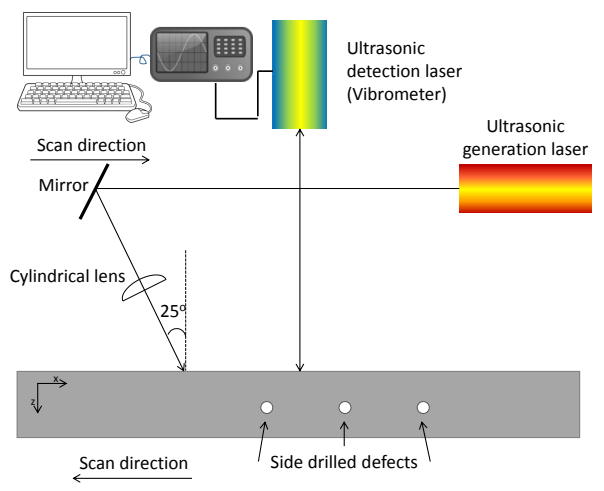

Fig. 2. Schematic of the experimental setup used. 
The experimental setup is shown in Fig. 2. A Nd:YAG laser (Picolo MOPA by Innolas), emitting at $1064 \mathrm{~nm}$ wavelength with a pulse duration of $1 \mathrm{~ns}$ and a pulse repetition rate of $5 \mathrm{KHz}$, was used as the ultrasonic generation laser. Its average power was $530 \mathrm{~mW}$, as measured in front of the sample, corresponding to $100 \mu \mathrm{J}$ per pulse. The laser beam was focused onto a line with approx. $5 \mathrm{~mm}$ length and 0.2 $\mathrm{mm}$ width. The incidence angle was approx. $25^{\circ}$ to facilitate scanning. A Polytech vibrometer (OFV-534 head with OFV5000 controller) was used to detect the ultrasonic signal. The light of the $633 \mathrm{~nm} \mathrm{CW} \mathrm{HeNe} \mathrm{laser} \mathrm{that} \mathrm{it} \mathrm{emits} \mathrm{had} \mathrm{a} \mathrm{power}$ of $<1 \mathrm{~mW}$, was focused to a $0.04 \mathrm{~mm}$ diameter spot and was aligned with the middle of the generation line source with an angle of incidence $0^{\circ}$ with respect to the normal.

In order to synthesize the LIPA, the generation laser was scanned across all consecutive element positions, while the detection laser remained focused at one position. Then the sample was moved to another element position, consequently the detection laser now irradiated another element position and the generation laser was scanned again across all element positions (see Fig. 2).

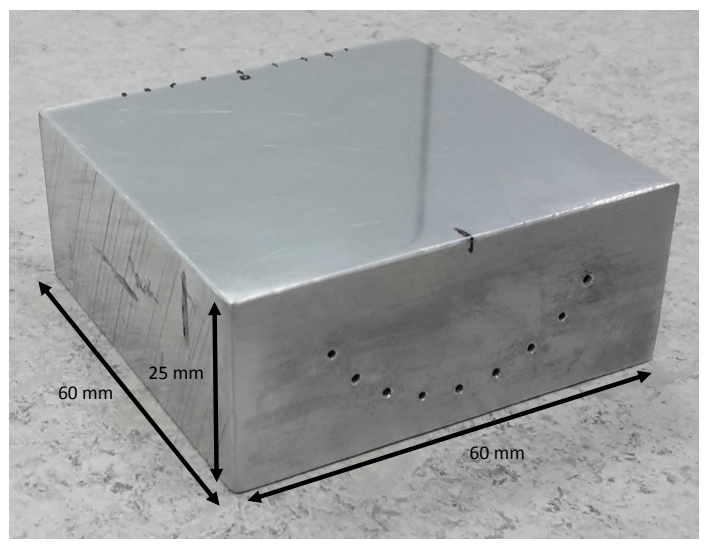

Fig. 3. Photo of the aluminum sample used.

An aluminum sample was used in the study. Its dimensions were $60 \times 25 \times 60 \mathrm{~mm}^{3}$. The sample had 9 defects (side through holes of diam. $1 \mathrm{~mm}$ ) arranged in a radial distribution, of radius $20 \mathrm{~mm}$ from the surface (see Fig. 3), in angles of $0^{\circ}, \pm 15^{\circ}, \pm 30^{\circ}, \pm 45^{\circ}, \pm 60^{\circ}$ from the surface normal. The side of the sample, where the laser beams were incident, was polished to a mirror surface, in order to maximize the light reflected to the vibrometer.

A 161 element LIPA, with element spacing of $155 \mu \mathrm{m}$ was synthesized, with the array center coinciding with the center of the defects' radial distribution. The bandwidth of the vibrometer was DC to $24 \mathrm{MHz}$ and a $1 \mathrm{MHz}$, high pass, and a $21 \mathrm{MHz}$ low pass, analogue filters were used while capturing the signal. Each captured waveform was averaged 500 times.

\section{B. Results}

During the post processing, digital filtering of the signal was performed, in order to maximize the SNR, using a Gaussian filter with $100 \%$ bandwidth, at $-40 \mathrm{~dB}$. The band pass digital filters applied had various central frequency values as specified in each case.

Fig. 4, 5, 6 show the effect of apodization at the TFM image. It can be seen that the apodized images show better SNR either normalized or not. The normalization enhances the imaging in blind spots, i.e. areas of low sensitivity, in the image. This set of figures is a multi mode analysis using TFM images [11]. It can be seen that different modes are more sensitive to detecting defects at different angles. Information from all modes can be combined to detect defects from $0^{0}$ to $60^{\circ}$.

\section{CONCLUSIONS}

This paper has presented improvements on the imaging algorithm used for post processing of LIPAs. The directivity and sensitivity patterns of laser ultrasonics have been considered in the expression for the TFM, introducing apodization. In this way, there is a more efficient use of the data of the full matrix. The experimental results show that the apodization maximizes the signal to noise ration (SNR) at each point in the image, facilitating defect detection. Laser ultrasonic generation excites simultaneously bulk and surface acoustic waves. Longitudinal, shear and mode converted bulk waves were used for ultrasonic images of the aluminum sample, applying a multi-mode TFM. The results show that using more than one modes and using appropriate digital frequency filtering for the imaging algorithm, detection of defects can be achieved in a wide range of angles $\left(0^{\circ}-60^{\circ}\right)$.

\section{REFERENCES}

[1] T. Stratoudaki, M. Clark, and P. D. Wilcox. Laser induced ultrasonic phased array using full matrix capture data acquisition and total focusing method. Opt. Express, 24(19):21921-21938, 2016.

[2] M. Dubois, M. Militzer, A. Moreau, and J. F. Bussiere. A new technique for the quantitative real-time monitoring of austenite grain growth in steel. Scripta Materialia, 42:867-874, 2000.

[3] K. R. Yawn, M. A. Osterkamp, D. Kaiser, and C. Barina. Improved laser ultrasonic systems for industry. In Review of Progress in Quantitative Nondestructive Evaluation, volume 1581, pages 397-404. AIP, 2014.

[4] L. S. Wang, J. S. Steckenrider, and J. D. Achenbach. A fiberbased laser ultrasonic system for remote inspection of limited access components. In Review of Progress in Quantitative Nondestructive Evaluation, volume 16, pages 507-514. Plenum Press, 1997.

[5] J. R. Bernstein and J. B. Spicer. Line source representation for lasergenerated ultrasound in aluminum. J. Acoust. Soc. Am., 107:1352-1357, 2000.

[6] M.-H. Noroy, D. Royer, and M. Fink. Shear-wave focusing with a laser-ultrasound phased-array. IEEE Trans. Ultrason., Ferroelectr., Freq. Control, 42:981-988, 1995.

[7] G. F. Miller and H. Pursey. The field and radiation impedance of mechanical radiators on the free surface of a semi-infinite isotropic solid. In Proceedings of the Royal Society of London. Series A, Mathematical and Physical Sciences, volume 223, pages 521-542. Royal Society, 1954.

[8] J. Zhang, B. W. Drinkwater, and P. D. Wilcox. Defect characterization using an ultrasonic array to measure the scattering coefficient matrix. IEEE Trans. Ultrason., Ferroelectr., Freq. Control, 55:2254-2265, 2008.

[9] B. W. Drinkwater and P. D. Wilcox. Ultrasonic arrays for non-destructive evaluation: A review. NDT \& E International, 39(7):525-541, 2006.

[10] A. McNab, A. Cochran, and M. A. Campbell. The calculation of acoustic fields in solids for transient normal surface force sources of arbitrary geometry and apodization. J. Acoust. Soc. Am., 87(4):1455-1465, 1990.

[11] J. Zhang, B. W. Drinkwater, P. D. Wilcox, and A. J. Hunter. Defect detection using ultrasonic arrays: The multi-mode total focusing method. NDT \& E International, 43:123-133, 2010. 


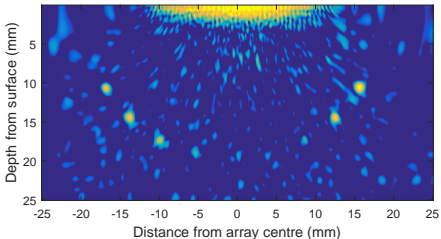

(a)

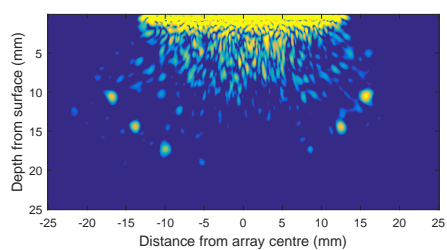

(d)

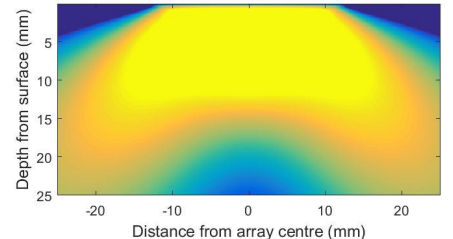

(b)

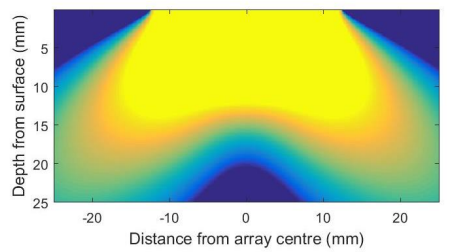

(e)

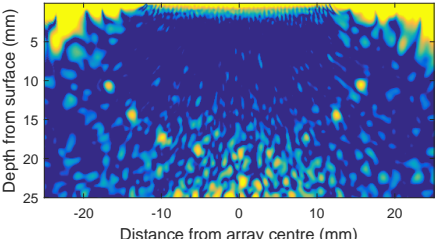

(c)

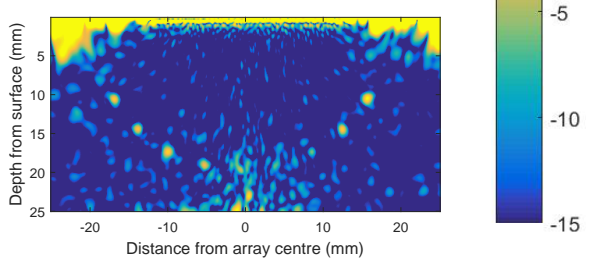

(f)

(g)

Fig. 4. TFM images of aluminum sample using longitudinal-longitudinal mode and a $6 \mathrm{MHz}$ digital filter, (a), (b), (c) without apodization terms and (d), (e), (f) with apodization terms. (a) and (d) are TFM images with uniform noise and non-uniform sensitivity. (b) and (e) are images of the sensitivity as described in Eq. (6), without and with the apodization terms respectively. (c) and (f) are normalized TFM images as described in Eq. (7). The dynamic range is also shown in $(\mathrm{g})$

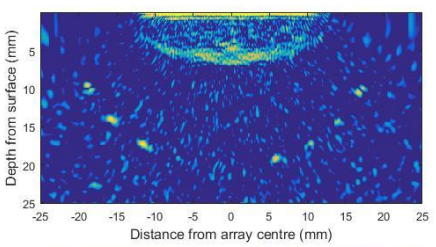

(a)

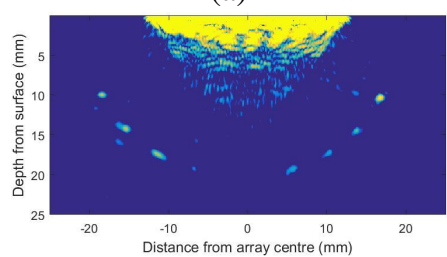

(d)

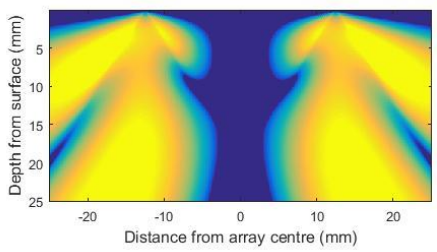

(b)

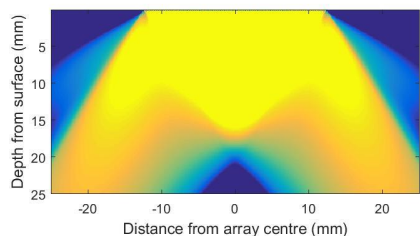

(e)

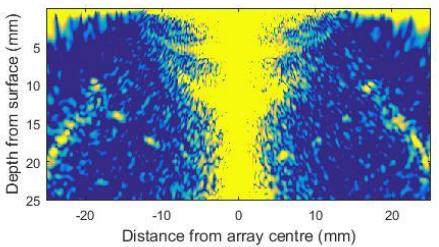

(c)

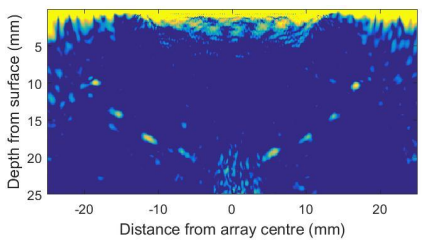

(f)

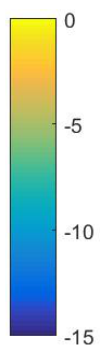

(g)

Fig. 5. TFM images of aluminum sample using shear-shear mode and a $5 \mathrm{MHz}$ digital filter. The individual graphs are presented in the same order as Fig. 4

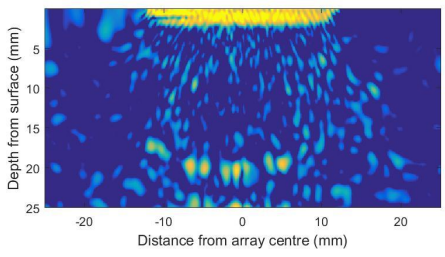

(a)

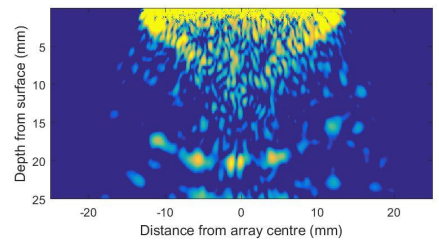

(d)

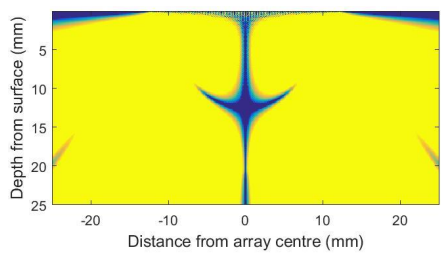

(b)

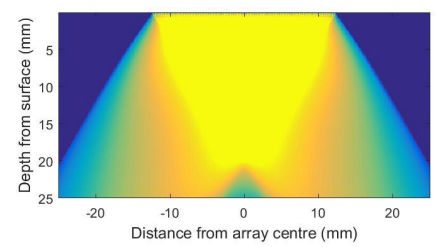

(e)

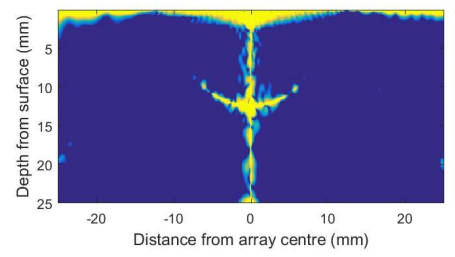

(c)

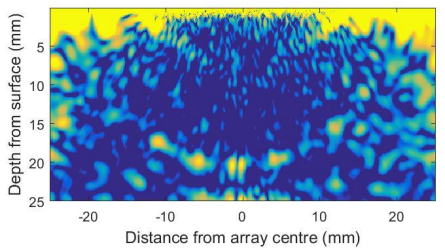

(f)

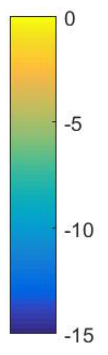

(g)

Fig. 6. TFM images of aluminum sample using shear-longitudinal mode and a $3 \mathrm{MHz}$ digital filter. The individual graphs are presented in the same order as Fig. 4 